\title{
Caracterización y extracción lipídica de las semillas del cacao amazónico (Theobroma grandiflorum)
}

\author{
Characterization and Lipid Extraction of Amazon Cocoa Seeds (Theobroma \\ grandiflorum)
}

\author{
E. Alviárez G. ${ }^{\mathrm{a}}$ \\ W. Murillo A. ${ }^{\mathrm{a}}$ \\ E. Murillo P. ${ }^{\mathrm{a}}$ \\ B. A. Rojano ${ }^{\mathrm{b}}$ \\ J. J. Méndez A. ${ }^{\mathrm{a}, *}$
}

Recepción: 17-jun-2015

Aceptación: 20-ene-2016

\section{Resumen}

La pulpa de Theobroma grandiflorum, conocido comúnmente como copoazú, es utilizada para la preparación de mermeladas, jugos, yogurt, néctares y dulces, y sus semillas son aprovechadas para la elaboración de cupulate, bebida equivalente al chocolate. En Colombia existe poca información sobre las propiedades de este fruto, especialmente de los ácidos grasos presentes en las semillas, las cuales podrían ser de gran interés para la industria alimentaria. En este estudio se identificó y analizó el contenido de ácidos grasos presentes en el aceite extraído de las semillas de copoazú en madurez fisiológica y sus índices de calidad; además, se midió el potencial antioxidante del aceite mediante el ensayo ORAC; lo anterior como aproximación al conocimiento del uso potencial alimenticio o nutracéutico de uno de los frutos exóticos de la amazonia de poco conocimiento en Colombia. La identificación de ácidos grasos reveló que hay mayor contenido de ácido oleico $(36,3 \%)$, esteárico $(29,27 \%)$ y palmítico $(7,26 \%)$; los índices de calidad, como la saponificación (1,88,15 mg KOH/g muestra), yodo (49,33 g de I/g de grasa) y acidez (1,04 mg KOH/g muestra), muestran que el aceite de copoazú podría ser utilizado en la industria alimentaria y cosmética.

Palabras clave: ácidos grasos, copoazú, índices de calidad, potencial nutricional, Theobroma grandiflorum.

\begin{abstract}
The pulp from Theobroma grandiflorum, commonly known as Copoazú, is used to prepare jams, juices, yogurt, and nectars and sweets, while their seeds are exploited for the development of cupulate, a beverage which is the equivalent to chocolate. In Colombia, there are few reports about the properties of this fruit, especially about the fatty acids from its seeds, which could be of great interest in the food industry. Therefore, in this study were identified and analyzed the fatty acids content, which are present in the Copoazú seeds extracted oil, in its physiological maturity and analyzed its qualities indices. Also the fat antioxidant potential was measured by the ORAC essay. The above approach to understand the nourishing potential use of the

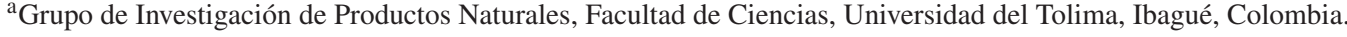
*Autor de correspondencia: jmendez@ut.edu.co

${ }^{\mathrm{b}}$ Laboratorio Ciencia de los Alimentos. Universidad Nacional de Colombia, Medellín, Colombia.
} 
food and/or nutraceutical, in one of the Amazon exotic fruits little known in Colombia. The identification of fatty acids showed that there is a high content of oleic (36.3\%), stearic $(29.27 \%)$ and palmitic acid $(7.26 \%)$; plus, the qualities indices like the saponification (1.88,15 mg KOH/g sample), iodine (49.33 $\mathrm{g} \mathrm{de} \mathrm{I} / \mathrm{g}$ oil) and acid (1.04 mg KOH/g sample) show that Copoazu oil could be used in food and cosmetic industries.

Key words: Fatty Acids, Nutritional Potential, Qualities Indices, Theobroma Grandiflorum.

\section{Introducción}

El Theobroma grandiflorum, conocido comúnmente en Colombia como copoazú, cacao blanco o cacao amazónico, es un árbol perenne propio del bosque húmedo tropical de la región amazónica, perteneciente a la familia esterculiácea. En la región se utiliza su pulpa para la preparación de mermeladas, jugos, yogurt, néctares y dulces, mientras que sus semillas son aprovechadas para la elaboración de 'cupulate' que es una bebida equivalente al chocolate [1]. No obstante, no existen suficientes reportes sobre las propiedades de los ácidos grasos de las semillas de este fruto amazónico, aspecto que es indispensable para promover su mayor aprovechamiento a nivel agroindustrial. Actualmente, el consumo de alimentos ricos en ácidos grasos ha ganado importancia, debido a que estos, y principalmente los poliinsaturados, tienen propiedades antioxidantes y antimicrobianas [2] y contribuyen a la reducción de enfermedades como la diabetes tipo 2 [3].

Las investigaciones se han centrado en la determinación de las propiedades de los aceites extraídos de semillas de diferentes frutos, como el píritu (Bactris piritu) [4], el corozo (Acrocomia aculeata Jacq.) [5], el coquito (Jubaea chilensis) [6], el pequi (Caryocar brasiliense, camb.) [7], la sandía (Citrullus lanatus) [8], el albaricoque (Prunus armeniaca L.) [9] y la calabaza (Cucurbita moschata) [10]. En cuanto a la grasa extraída de las semillas de copoazú, los estudios se han enfocado en el conocimiento de la composición de sus ácidos grasos y en la determinación de las propiedades reológicas en mezclas con la manteca de cacao [11,12].

En los estudios realizados al copoazú se ha determinado que la pulpa tiene potencial antioxidante [13,14], y en las semillas, Yang et al. [15] identificaron proantocianidinas y flavonoides como quercetina y caemferol, compuestos que han sido relacionados con la disminución del riesgo de padecer ciertas enfermedades.
Por estas razones, el objetivo de este trabajo fue identificar la composición de los ácidos grasos presentes en el aceite de las semillas de copoazú, evaluar la calidad de este y determinar su capacidad antioxidante, mediante el ensayo ORAC, correlacionándola con el contenido fenólico de las semillas.

\section{Materiales y métodos}

Material vegetal. Las semillas de copoazú (Theobroma grandiflorum) se obtuvieron de frutos maduros recolectados en el municipio de Miraflores, en el departamento del Guaviare $\left(1^{\circ} 20^{\prime} 12^{\prime \prime} \mathrm{N}\right.$ $71^{\circ} 57^{\prime} 04^{\prime \prime} \mathrm{O}$ ), perteneciente a la zona de bosque húmedo tropical, en el mes de septiembre del 2011. Los frutos fueron transportados vía terrestre hasta la Universidad del Tolima, en la ciudad de Ibagué, donde las semillas fueron extractadas manualmente.

Reactivos. Trolox (6-hidroxi-2, 5, 7, 8-tetrametilcroman-2-carboxílico), n-hexano, etanol, reactivo de Folin Ciocalteu, ácido gálico y fluoresceína; fueron comprados a Aldrich Chem. Co y Merck (Alemania).

Preparación de extractos. Las semillas se secaron durante dos días a una temperatura de $50{ }^{\circ} \mathrm{C}$; posteriormente, fueron trituradas $(2 \mathrm{~mm})$ y extraídas con etanol (1:10) en un equipo soxhlet [16]. El extracto obtenido se filtró con papel whatman 1, se concentró a presión reducida y fue almacenado a 4 ${ }^{\circ} \mathrm{C}$ hasta su uso en los diferentes ensayos.

Extracción del aceite. La extracción del aceite de las semillas de copoazú en madurez fisiológica se realizó por el método soxhlet, utilizando n-hexano como solvente, durante dos horas, a una temperatura promedio de $68^{\circ} \mathrm{C}$, y posteriormente se evaporó el solvente a presión reducida en un rotaevaporador y se almacenó en frasco ámbar protegido de la luz. 
Análisis de ácidos grasos. La identificación se llevó a cabo por cromatografía de gases-espectrometría masas (GC-MS, AGILENT 6890N acoplado a detector de masas AGILENT TECHNOLOGIES NETWORK 5973 Mass Selective Detector, Columna capilar 5\% difenil, $95 \%$ dimetilpolisiloxano, WCOT Fused Silica VF-5 ms Varían $30 \mathrm{~m}$ x 0,25 mm x $0,25 \mu \mathrm{m}$, Inyector: Gas portador: Helio, Temperatura: $280{ }^{\circ} \mathrm{C}$ Horno: Temperatura inicial: $100{ }^{\circ} \mathrm{C}$; Temperatura Final: $280{ }^{\circ} \mathrm{C}$; Duración Total: 53,50 minutos; Detector de masas Modo d- adquisición: scan; Rango de masas bajo: 40, Rango de masas alto: 600). La identificación se realizó mediante la comparación con los espectros de masa con la librería Wiley (2010), usando como patrón estándar FAME (ácidos grasos ésteres metílicos) y se establecieron índices de similaridad superiores al $90 \%$.

Índices de calidad del aceite. Según las normas establecidas por la A.O.A.C, se determinaron los índices de refracción (A.O.A.C Cc 7-25, 2009), la densidad (A.O.A.C. Cc 10c-95, 2009), la acidez (A.O.A.C Cd 3d-63, 2009), la saponificación (A.O.A.C Cd 3-25, 2003) y el yodo (A.O.A.C $993,20,1993)$. Todas las pruebas se realizaron con aceite fresco para evitar la oxidación o degradación del aceite.

Determinación de dienos conjugados. Los dienos conjugados en el aceite de copoazú se determinaron según la metodología descrita por Zuta [17], con algunas modificaciones, diluyendo la muestra en n-Hexano ( $1 \% \mathrm{p} / \mathrm{v})$, a diferentes temperaturas (25-27 ${ }^{\circ} \mathrm{C}, 37{ }^{\circ} \mathrm{C}, 50{ }^{\circ} \mathrm{C}$ y $70{ }^{\circ} \mathrm{C}$ ), y se midieron por espectrofotometría absorbancias entre 0,2 y 0,8 a $232 \mathrm{~nm}$. Los resultados se expresaron como $\varepsilon(\mathrm{cm})^{-1}$.

Actividad antioxidante. El procedimiento experimental se basó en reportes previos de Ou y colaboradores [18] y Litescu y colaboradores [19], emplea TROLOX ${ }^{\circledR}$. Como estándar y condiciones controladas de temperatura a $37^{\circ} \mathrm{C}$ y pH 7,4. Las lecturas se realizaron a una $\lambda$ de excitación $493 \mathrm{~nm}$ y split de excitación 10, $\lambda$ de emisión $515 \mathrm{~nm}$ y slit de emisión 15 , con atenuador del $1 \%$. Para el desarrollo de la técnica se utilizaron soluciones de fluoresceína $1 \times 10^{-2}$ M y AAPH 0,6 M en buffer fosfato (75 mM, pH 7,4). Una alícuota de $30 \mu \mathrm{L}$ muestra se mezcló con $21 \mu \mathrm{L}$ de fluoresceína, 2,899 $\mu \mathrm{L}$ de buffer fosfato y $50 \mu \mathrm{L}$ de AAPH. El efecto protector del antioxidante se calculó usando las diferencias de áreas bajo la curva de decaimiento de la fluoresceína entre el blanco y la muestra, y se comparó con la curva patrón; los resultados se expresaron en micromoles equivalentes de Trolox por litro de muestra ( $\mu$ ol Trolox/L de muestra), ecuación (1).

$$
O R A C=\frac{A U C-A U C^{0}}{A U C_{\text {trolox }}-A U C^{0}} f \text { Trolox }
$$

Donde AUC es el área bajo la curva de la muestra; $\mathrm{AUC}^{\circ}$, el área bajo la curva para el control; AUCTrolox, el área bajo la curva para el Trolox, y f, el factor de dilución de los extractos. La fluorescencia se midió en un espectrofotómetro de fluorescencia (PerkinElmer ${ }^{\circledR}$ LS55). Estos parámetros fueron analizados por el software OriginPro ${ }^{\circledR}$ (Origin Lab, Northampton, MA).

Análisis estadístico. Para todos los análisis se realizaron cuatro réplicas. Los datos fueron reportados como la media de cuatro determinaciones $(n=4 \times 4)$ DE $(4 \times 4)$, mediante el uso del programa estadístico InfoStat/Profesional ${ }^{\circledR}$.

\section{Resultados}

\subsection{Identificación de los ácidos grasos}

En la tabla 1 se muestran los porcentajes de los ácidos grasos identificados en el aceite extraído de las semillas de copoazú; se lograron identificar el $90 \%$ de los compuestos de la muestra, encontrándose en mayor proporción los ácido oléico (36,30\%) y el ácido esteárico $(29,27 \%)$, conformando los dos el $65.57 \%$ del material oleícola; importante tener en cuenta que el 50,43\% del aceite de copoazú lo constituyen ácidos grasos de tipo saturado, como el araquídico $(11,22 \%)$ y el palmítico $(7,26 \%)$, también se reporta el ácido linoleico, aunque en cantidad relativamente baja $(3,56 \%)$.

Al compararse con la manteca de cacao (tabla 1), el aceite de copoazú exhibe ácidos grasos similares, en distintas proporciones; en el caso del ácido graso palmítico y esteárico, se presenta menor contenido, mientras que aparece un mayor nivel de ácido oléico $(36,30 \%)$, linoléico $(3,60 \%)$ y araquídico $(11,22 \%)$. 
Tabla 1. Comparación entre la composición de ácidos grasos de aceite de copoazú y cacao.

\begin{tabular}{lcccc}
\hline \multicolumn{1}{c}{ Ácidos grasos } & & $\begin{array}{c}\text { Copoazú } \\
\mathrm{m} \mathrm{p} / \mathrm{p}(\%)\end{array}$ & $\begin{array}{c}\text { Cacao* } \\
\mathrm{m} \mathrm{p} / \mathrm{p}(\%)\end{array}$ & $\begin{array}{c}\text { Copoazú* } \\
\mathrm{m} \mathrm{p} / \mathrm{p}(\%)\end{array}$ \\
\hline ácido cáprico & $\mathrm{C} 10: 0$ & $0,01 \pm 0,00$ & $\mathrm{NR}$ & $\mathrm{NR}$ \\
ácido láurico & $\mathrm{C} 12: 0$ & $0,01 \pm 0,00$ & $\mathrm{NR}$ & $\mathrm{NR}$ \\
ácido tridecanóico & $\mathrm{C} 13: 0$ & $0,02 \pm 0,00$ & $\mathrm{NR}$ & $\mathrm{NR}$ \\
ácido mirístico & $\mathrm{C} 14: 0$ & $0,03 \pm 0,02$ & 0,1 & $\mathrm{NR}$ \\
ácido pentadecanóico & $\mathrm{C} 15: 0$ & 0,00 & $\mathrm{NR}$ & $\mathrm{NR}$ \\
ácido palmítico & $\mathrm{C} 16: 0$ & $7,26 \pm 0,20$ & 26,8 & 7,8 \\
ácido palmitoléico & $c 9-\mathrm{C} 16: 1$ & $0,12 \pm 0,04$ & 0,3 & 0,1 \\
ácido margárico & $\mathrm{C} 17: 0$ & $0,20 \pm 0,01$ & 0,2 & 0,2 \\
ácido heptadecenóico & $c 10-\mathrm{C} 17: 1$ & $0,01 \pm 0,00$ & $\mathrm{NR}$ & $\mathrm{NR}$ \\
ácido esteárico & $\mathrm{C} 18: 0$ & $29,27 \pm 0,80$ & 33,6 & 32,9 \\
ácido oleico & $c 9-\mathrm{C} 18: 1$ & $36,30 \pm 1,00$ & 34,9 & 42,2 \\
ácido linoelaidico & $t 9,12-\mathrm{C} 18: 2$ & $0,04 \pm 0,00$ & $\mathrm{NR}$ & $\mathrm{NR}$ \\
ácido linoleico & $c 9,12-\mathrm{C} 18: 2$ & $3,60 \pm 0,08$ & 2,9 & 3,5 \\
ácido araquídico & $\mathrm{C} 20: 0$ & $11,22 \pm 0,32$ & 1,0 & 9,8 \\
ácido $\alpha$ linolénico & $c 9,12,15-\mathrm{C} 18: 3(\mathrm{n}-3)$ & $0,20 \pm 0,01$ & 0,2 & 0,2 \\
ácido gadoleico & $c 11-\mathrm{C} 20: 1$ & $0,35 \pm 0,01$ & $\mathrm{NR}$ & $\mathrm{NR}$ \\
ácido henicosanoico & $\mathrm{C} 21: 0$ & $0,04 \pm 0,00$ & $\mathrm{NR}$ & $\mathrm{NR}$ \\
ácido eicosadienoico & $c 11,14-\mathrm{C} 20: 2$ & $0,22 \pm 0,00$ & $\mathrm{NR}$ & $\mathrm{NR}$ \\
ácido behénico & $\mathrm{C} 22: 0$ & $2,11 \pm 0,06$ & $\mathrm{NR}$ & 3,2 \\
ácido lignocérico & $\mathrm{C} 24: 0$ & $0,25 \pm 0,01$ & $\mathrm{NR}$ & $\mathrm{NR}$ \\
\hline
\end{tabular}

*tomado Lannes, S., Medeiros, M., y Gioielli, L. (2004).

NR: No reportado

Tabla 2. Índices de calidad del aceite de copoazú.

\begin{tabular}{lccc}
\hline Parámetros & Aceite de copoazú & Manteca de cacao & Rango Codex \\
\hline Densidad $^{a}$ & $0,88 \pm 0,1$ & $0,856-0,86$ & $0,9-0,96$ \\
Índice de refracción $^{\text {Índice de acidez }}{ }^{b}$ & 1,38 & $1,453-1,457$ & \\
Índice de saponificación $^{b}$ & $1,04 \pm 0,1$ & 1,75 & 4,00 \\
Índice de Yodo $^{c}$ & $488,15 \pm 1,5$ & $190-200$ & $184-196$ \\
\hline
\end{tabular}

${ }^{a} \mathrm{~g} / \mathrm{mL} ;{ }^{b} \mathrm{mg} \mathrm{KOH} / \mathrm{g}$ muestra; ${ }^{c} \mathrm{~g}$ de $\mathrm{I} / \mathrm{g}$ de aceite.

Se contrastó también con el aceite de copoazú de la región brasilera, y se evidenció que en ambas hay mayor proporción de los ácidos grasos esteárico, palmítico, oléico, encontrándose que tienen valores similares. ¿Cuál es la distribución de los ácidos grasos en el triacilglicerol: SOS, SOP?; esta configuración determina las propiedades de plasticidad, textura y estabilidad química de la grasa.

\section{2. Índices de calidad}

En la tabla 2 se presentan los valores de la densidad y el índice de refracción del aceite crudo de copoazú comparado con la manteca de cacao; de igual manera, la tabla muestra los rangos normados según el Codex Alimentarium (1999). Se puede apreciar que la densidad y el índice de refracción de la grasa de interés en este trabajo muestran valores por debajo de los rangos aceptados por la Norma Técnica Colombiana NTC 574 (2008) para la manteca de cacao, debido a que el nivel de insaturaciones que presenta es bajo, como se puede verificar en la tabla 1. En la tabla 2 también se observa que el aceite de copoazú presenta un bajo índice de acidez, comparado con la manteca de cacao; esto lo haría menos predispuesto a la degradación oxidativa, por la baja presencia de ácidos grasos libres. En cuanto al índice de saponificación, este resultó cercano al rango mínimo de la manteca de cacao, explicable por la coincidente composición en algunos ácidos grasos; la proporción en la que estos aparecen es lo que las diferencia (tabla 2).

Uno de los atributos de un aceite asociados directamente al grado de insaturación de sus ácidos grasos es el índice de yodo, lo que se corrobora al observar la información reportada en la tabla 1. Mientras que la aceite de copoazú está constituída por un 50,43\% de ácidos grasos saturados y 40,82\% de insaturados, 
la manteca de cacao contiene $69,3 \%$ de ácidos grasos saturados y $30,7 \%$ de insaturados [20].

La capacidad del aceite de copoazú para resistir los efectos de la energía radiante (UV), calórica o enzimática se determinaron a través del índice de peróxido y dienos conjugados. En la tabla 3 se pueden apreciar las condiciones empleadas en estos ensayos. Claramente se observa cómo los incrementos de temperatura duplican o modifican hasta veinte veces el índice de peróxidos. A pesar de que el índice de dienos no sufre alteraciones tan significativas, se nota, igualmente, la incidencia de la temperatura sobre los valores obtenidos.

Tabla 3. Medición de la rancidez oxidativa con respecto a la temperatura.

\begin{tabular}{ccc}
\hline \multicolumn{1}{c}{ Parámetros } & Valores \\
\hline Índice de peróxidos $^{a}$ & $25-27^{\circ} \mathrm{C}$ & $2,98 \pm 0,0$ \\
& $50{ }^{\circ} \mathrm{C}$ & $24,91 \pm 3,7$ \\
& $70^{\circ} \mathrm{C}$ & $52,81 \pm 03$ \\
Índice de dienos conjugados $^{b}$ & $25^{\circ}-27^{\circ} \mathrm{C}$ & $0,77 \pm 0,0$ \\
& $37^{\circ} \mathrm{C}$ & $1,24 \pm 0,01$ \\
& $50{ }^{\circ} \mathrm{C}$ & $1,38 \pm 0,03$ \\
& $70{ }^{\circ} \mathrm{C}$ & $1,50 \pm 0,0$ \\
\hline
\end{tabular}

${ }^{a}$ meq de $\mathrm{O}_{2} / \mathrm{kg} ;{ }^{b} \varepsilon(\mathrm{cm})^{-1}$

\section{Actividad antioxidante}

La capacidad antioxidante del aceite de copoazú, medida a través del ensayo de capacidad de absorción del radical oxígeno (ORAC), presentó mejor potencial antioxidante que el reportado para la pulpa de la fruta $(31,71 \pm 15,1$ micromoles equivalentes de Trolox/g de fruta) [14]; sin embargo, su actividad estabilizadora del radical peroxilo $(140,32 \pm 1,3$ micromoles equivalentes de Trolox/g de aceite) es moderada, comparada con el aceite de coco [2], debido, posiblemente, a su bajo contenido de tocoferoles y carotenos, lo que lo hace propenso a tener un alto grado de oxidación, contrario a lo comentado anteriormente; aspecto que puede ser consecuencia de la degradación del aceite en el momento de realizar el test.

Debe tenerse presente que los ácidos grasos insaturados, particularmente los poliinsaturados, tienen mayor predisposición a la oxidación que los saturados, observándose una baja resistencia a la oxidación; esto puede ser debido a una escasa presencia de compuestos antioxidantes naturales, como son los compuestos fenólicos, carotenoides o tocoferoles en el aceite, lo que se corrobora al observar el valor obtenido en el ensayo ORAC.

\section{Discusión}

\subsection{Caracterización del aceite de cacao amazónico colombiano}

Las particularidades encontradas en el contenido de ácidos grasos del aceite de copoazú de origen colombiano y de la región brasilera son explicables por la diferencia composicional del terreno en el que se cultiva y por la disparidad climática o genética, entre otros [21]. El aceite de origen colombiano presenta un buen contenido de ácidos grasos, lo cual favorece su industrialización como base de productos como champú, jabones y cremas, y constituye una oportunidad de ampliación de cultivos en la zona de la Amazonía colombiana, generando una fuente económica para los cultivadores de esa zona. El bajo índice de acidez que exhibe este aceite de copoazú constituye una bondad adicional, factor que pudo ser influido por la temperatura utilizada en la extracción, 60-70 ${ }^{\circ} \mathrm{C}$, rango en el cual se considera que las lipasas encargadas del aumento de ácidos grasos libres se inactiven [22].

Considerando que el índice de saponificación está asociado a la longitud de la cadena [23], debe tenerse presente que el perfil lipídico que muestra el material graso del copoazú colombiano podría sustentarse no solo en este parámetro, sino también en la variabilidad de ácidos grasos y la proporcionalidad relativa en que se encuentran en el producto. Según la norma Codex 1999, el aceite de copoazú se clasifica como un aceite no secante, lo que la habilita para ser utilizado en la industria cosmética [23].

Cabe mencionar que los bajos valores del índice de refracción y de la densidad encontrados en el aceite de copoazú estarían asociados al mayor número de insaturaciones en la cadena carbonada de los ácidos grasos, otorgándole mayor viscosidad al aceite [24] e incrementando, consecuentemente, el índice de refracción y la densidad.

De otra parte, se sabe que la degradación oxidativa inicia con el ataque de EROS y continúa rápidamente con la formación de un radical lipoperóxido (LOO•); cuando este remueve hidrógenos de un ácido graso insaturado o poliinsaturado se generan hidroperóxidos (LOOH) y, paralelamente, dienos conjugados 
[25]; todo lo anterior incide en las características sensoriales del aceite y desmejora consecuentemente su uso y calidad. Una consecuencia de importancia relevante relacionada con la oxidación de los ácidos grasos es la formación intracelular de las células de espuma, signo inconfundible de la presencia de colesterol asociado a LDL, relacionado con la generación de lesiones ateroscleróticas tempranas [26]. Es importante tener en cuenta que pequeñas cantidades de hidroperóxidos en un aceite pueden tener origen por la acción de las lipoxigenasas en el vegetal, mucho antes de la extracción del aceite [27].

\section{Conclusiones}

Los resultados muestran que el aceite de copoazú tiene propiedades antioxidantes, que es una fuente importante de ácidos grasos oléico, araquídico y linoleico y que, además, presenta propiedades que le permiten ser utilizado industrialmente en el área alimentaria o cosmética.

\section{Agradecimientos}

Al Laboratorio LASEREX de la Universidad del Tolima, al Laboratorio Ciencia de los Alimentos de la Universidad Nacional de Colombia, sede Medellín, y a la Universidad de Lleida (España), por el apoyo logístico.

\section{Referencias}

[1] S. Rojas, J. Zapata, E. Pereira y E. Varon, El cultivo del copoazú (Theobroma grandiflorum) en el piedemonte amazónico colombiano. Corpoica, Convenio de Cooperación Gobierno de Colombia- Unión Europea, 1998.

[2] G. A. Bataglion, F M. da Silva, J. M. Santos, F. N. dos Santos, M. T. Barcia, C. C. de Lourenço, M. Salvador, H. Godoy, M. Eberlin, and H. H. Koolen, "Comprehensive characterization of lipids from Amazonian vegetable oils by mass spectrometry techniques", Food Research International, vol. 64, pp. 472-481, 2014.

[3] M. B. MacLenan, S. E. Clarke, K. Pére, G. A. Madera, W. J. Muller, J. X. Kang, and D. W. $\mathrm{Ma}$, "Mammary tumor development is directly inhibited by lifelong $n-3$ polyunsaturated fatty acids", The Journal of nutritional biochemistry, vol. 24, no. 1, pp. 388-395, 2013.
[4] C. Belén, I. López, J. Barranco, D. García, M. Moreno y O. Linares, "Caracterización fisicoquímica del aceite de la semilla de Píritu (Bactris piritu (H. Karst) H. Wendl)". Grasas y Aceites, vol. 55, no. 2, pp. 138-142, doi: 10.3989/gya.2004.v55.i2.158, 2004.

[5] B. Camacho, I. López, D. García, M. González, M. Moreno-Álvarez y C. Medina, "Evaluación fisico-química de la semilla y del aceite de corozo (Acrocomia aculeata Jacq.)". Grasas y aceites, vol. 56, no. 4, pp. 311-316, 2005.

[6] L. Masson, C. Camilo y E. Torija. "Caracterización del aceite de coquito de palma chilena (Jubaea chilensis)". Rev. Grasas y aceites, vol. 59, no. 1, pp. 33-38, doi: 10.3989/gya.2008.v59.i1.487 (2008).

[7] L. P. Aquino, S. V. Borges, F. Queiroz, R. Antoniassi, ans M. A. Cirillo, "Extraction of oil from pequi fruit (Caryocar Brasiliense, Camb.) using several solvents and their mixtures", Grasas y Aceites, vol. 62, no. 3, pp. 245-252, 2011.

[8] S. Raziq, F. Anwar, Z. Mahmood, S. A. Shahid, and R. Nadeem, "Characterization of seed oils from different varieties of watermelon Citrullus lanatus (Thunb.) from Pakistan", Grasas y aceites, vol. 63, no. 4, pp. 365-372, 2012.

[9] M. Manzoor, F. Anwar, M. Ashraf, and K. M. Alkharfy, "Physico-chemical characteristics of seed oils extracted from different apricot (Prunus armeniaca $L$.) varieties from Pakistan", Grasas y aceites, vol. 63, no. 2, pp. 193-201, 2012.

[10] Z. Y. Petkova, and G. A. Antova, "Changes in the composition of pumpkin seeds (Cucurbita moschata) during development and maturation", Grasas y Aceites, vol. 66, no. 1, e058, 2015.

[11] S. Lannes, M. Medeiros, and L. Gioielli. "Rheological properties of cupuassu and cocoa fats", Grasas y Aceites, vol. 55, no. 2, pp. 115-121, doi: 10.3989/gya.2004.v55.i2.154, 2004.

[12] L. Quast, V. Luccas, and T. Kieckbusch, "Physical properties of pre-crystallized mixtures of cocoa butter and cupuassu fat", Grasas y Aceites, vol. 62, no. 1, pp. 62-67, doi: 10.3989/gya.034010, 2011. 
[13] E. Kuskoski, A. Asuero, A. Troncoso, J. Mancini-Filho y R. Fett, "Aplicación de diversos métodos químicos para determinar actividad antioxidante em pulpa de frutos", Cienc. Tecnol. Aliment., vol. 25, pp. 726-732, 2005.

[14] E. Alviárez, J.J. Mendez, B. Rojano, W. Murillo y C. Stringheta, "Poster Avaliação da atividade antioxidante da polpa, identificação dos ácidos graxos e propriedades da gordura do cupuaçu (Theobroma grandiflorum)", Simposio Latinoamericano de Ciência de Alimentos, Ciencia de Alimentos: Impacto na nutrição e saúde, 2013.

[15] H. Yang, P. Protiva, B. Cui, C. MA, S. Baggett, and E. Hequet, "New Bioactive Polyphenols from Theobroma grandiflorum ("Cupuacü")", Journal of Natural Products, vol. 66, no. 11, pp. 1501-1504, 2003.

[16] E. Murillo y J. J. Méndez, Guía metodológica para la detección rápida de algunos núcleos secundarios y caracterización de una droga cru$d a$, Universidad del Tolima, Facultad de Ciencias, Departamento de Química, GIPRONUT, 2011.

[17] P. Zuta, B. Simpson, X. Zhao, and L. Leclerc, "The effect of $\alpha$-tocopherol on the oxidation of mackerel oil", Food Chem., vol. 100, no. 2, pp. 800-807, 2007.

[18] B. Ou, D. Huang, M. Hampsch-Woodill, J. Flanagan, and E. Deemer, "Analysis of antioxidant activities of common vegetables employing oxygen radical absorbance capacity (ORAC) and ferric reducing antioxidant power (FRAP) assays: a comparative study", Journal of Agricultural and Food Chemistry, vol. 50, no. 11, pp. 3122-3128, 2002.

[19] S. Litescu, S. Eremia, A. Tache, I. Vasilescu, and G. Radu, "The Use of Oxygen Radical Absorbance Capacity (ORAC) and Trolox Equivalent Antioxidant Capacity (TEAC) Assays in the Assessment of Beverages' Antioxidant Properties", en Processing and impact on anti- oxidants in beverages, Capítulo 25, p. 245-249, 2014.

[20] L.M. Melgarejo, M. S. Hernández, J. A. Barrera y M. Carrillo, Oferta y potencialidades de un banco de germoplasma del género Theobroma en el enriquecimiento de los sistemas productivos de la región amazónica, Instituto Amazónico de Investigaciones Científicas "SINCHI", p. 157, 2006.

[21] J. Sánchez, E. Osorio, A. Montaño y M. Martínez, "Estudio del contenido em ácidos grasos de aceites monovarietales elaborados a partir de aceitunas producidas en la región extremeña", Grasas y Aceites, vol. 54, no. 4, pp. 371-377, 2003.

[22] D. Rozo y L. Velasco, Automatización del proceso de esterilización en la extracción de aceite de palma africana, Instituto de Investigación y Desarrollo de Tecnologías Aplicadas, IIDTA, Universidad de Pamplona, Pamplona, Colombia, 2007.

[23] J. Lafont, M. Páez, and A. Portacio, A., "Extracción y caracterización fisicoquímica del aceite de la semilla (almendra) del marañón (Anacardium occidentale L)", Información Tecnológica, vol. 22, no. 1, pp. 51-58, 2011.

[24] J. Santos, I. Santos, and A. Souza, "Effect of heating and cooling on rheological parameters of edible vegetable oils", Journal of Food Engineering, vol. 67, no. 4, pp. 401-405, 2005.

[25] S. Mennickent, M. Bravo, C. Calvo y M. Ave1lo, "Efectos pleiotrópicos de las estatinas", Rev. méd. Chile, vol. 136, no. 6, pp.) 775-782, 2008.

[26] J. Liao, and U. Laufs, "Pleiotropic effects of statins", Annu Rev Pharmacol Toxicol, vol. 45, pp. 89-118, 2005.

[27] M. Gordon, "The development of oxidative rancidity in foods". In: Antioxidants in food, Cambridge: CRC Press, pp. 10, 2001. 